\title{
Autoimmune Sera Reactive with Sm Antigen Contain High Levels of RNP-like Antibodies
}

\author{
Westley H. Reeves, David E. Fisher, Robert G. Lahita, and Henry G. Kunkel \\ The Rockefeller University, New York 10021
}

\begin{abstract}
Ribonucleoprotein particles containing Sm antigen were separated from particles containing both $\mathrm{Sm}$ and RNP antigens by ion-exchange chromatography to study the recognition of these antigens by autoimmune sera. By using the separated antigens, anti-Sm and/or anti-RNP antibodies were detected in $\sim 60 \%$ of sera from systemic lupus erythematosus patients by both enzyme-linked immunosorbent assay and immunoprecipitation of radiolabeled antigens followed by analysis on sodium dodecyl sulfate-polyacrylamide gels. These antibodies were detected in $30 \%$ of the same sera using the standard passive hemagglutination technique. Competition experiments demonstrated that all of the sera tested that contained anti-Sm antibodies also had anti-RNP-like reactivity. This latter reactivity usually represented $80 \%$ or more of the total $\mathrm{Sm}$ and $\mathrm{RNP}$ binding activity in lupus sera. The binding to RNP-like determinants by several of the sera was uniquely resistant to treatment of the antigen with snake venom exonuclease. These studies indicate that humoral immunity against $\mathrm{Sm}$ and RNP antigens in systemic lupus erythematosus is directed primarily against a single type of ribonucleoprotein particle in which the two antigens are physically associated. The specific binding to a single type of ribonucleoprotein particle suggests that this particle may be especially immunogenic and that it might play an important role in induction of the humoral immune response to Sm and RNP.
\end{abstract}

\section{Introduction}

Autoantibodies against nonhistone nuclear antigens are present in sera from many patients with systemic lupus erythematosus (SLE) ${ }^{1}$ and other connective tissue disorders (see Tan [1] for review). These autoantibodies are of interest because they can serve as markers for specific connective tissue disorders (1-4) and because sera containing these antibodies have been useful in characterizing a number of proteins involved in nuclear metabolism, including the Sm and RNP antigens (4-9). Antibodies to Sm are highly specific for SLE (2) and antibodies to RNP, although present in a variety of disorders including SLE $(1,10)$, are characteristically present in extremely high titers

Received for publication 23 March 1984 and in revised form 27 September 1984.

1. Abbreviations used in this paper: ELISA, enzyme-linked immunosorbent assay; ENA, extractable nuclear antigen; MCTD, mixed connective tissue disease; PAGE, polyacrylamide gel electrophoresis; PMSF, phenylmethylsulfonyl fluoride; SLE, systemic lupus erythematosus.

J. Clin. Invest.

(c) The American Society for Clinical Investigation, Inc.

0021-9738/85/02/0580/08 \$1.00

Volume 75, February 1985, 580-587 in sera from patients with mixed connective tissue disease (MCTD) $(11,12)$. Mattioli and Reichlin $(10,13)$ first showed that the Sm and RNP antigens are present on two types of particles, one containing $\mathrm{Sm}$ alone, and the other containing both Sm and RNP. These particles were separable by gel filtration and ion-exchange chromatography, and the RNP antigenic site was found to be sensitive to both ribonuclease (RNase) and trypsin, suggesting that the particles consist of both RNA and protein $(10,13,14)$. More recently, Lerner and Steitz (5) showed that Sm- and RNP-containing ribonucleoprotein particles contain a series of small nuclear RNA species (U1, U2, and U4-6) associated with eight proteins (termed bands A-G based upon their appearance on sodium dodecyl sulfate [SDS]-polyacrylamide gels) (5). RNP antigenic determinants are present on protein bands $\mathrm{A}$ and $\mathrm{C}$ in association with U1 RNA, whereas Sm determinants are found on the other six proteins (15-17) in association with $U 1$, U2, and U4-6 RNA.

The physical association of Sm and RNP antigens on U1-RNP particles and the difficulty in obtaining immunologically active RNP free of $\operatorname{Sm}(12,13)$ has made determination of the relative amounts of anti-Sm and RNP antibodies in sera difficult. In general, this has been accomplished by comparing the titers of antibody reactive with untreated ( $\mathrm{Sm}$ plus RNP) and RNase-treated (Sm) antigens, as in the hemagglutination assay (2). However, the existence of other RNasesensitive or resistant antigens in nuclear extracts (18), as well as the observation that $\mathrm{Sm}$ may be partially sensitive $(19,20)$ and RNP partly resistant (21) to RNase, make a more direct approach desirable. For this reason, we have attempted to distinguish between anti-Sm and anti-RNP antibodies using methods that are independent of the RNase sensitivity or resistance of the antigens to estimate more accurately the relative amounts of these antibodies in SLE and MCTD sera.

\section{Methods}

Patients. Serum was obtained from 50 consecutive patients seen in The Rockefeller University Hospital clinic who met the 1982 revised criteria of the American Rheumatism Association for SLE (22), from eight patients with clinical MCTD, and from 10 normal volunteers.

Detection of antibodies against nuclear antigens. Extractable nuclear antigens (ENAs) were prepared from rabbit thymus acetone powder (Pel Freeze Biologicals, Rogers, AR) (2) or from isolated nuclei (23) from human lymphoblastoid cell lines. Sm and RNP antibody titers were determined by passive hemagglutination as described by Tan and Peebles (2). The same antigen preparation was also used for determining the total antibody titer against ENAs ("anti-ENA antibody") by enzyme-linked immunosorbent assay (ELISA) as described (24). The wells of microtiter plates (Nunc, Vanguard International, Neptune, $\mathrm{NJ})$ were coated with crude rabbit thymus extract $(500 \mu \mathrm{g} / \mathrm{ml}$ total protein in $150 \mathrm{mM} \mathrm{NaCl}-10 \mathrm{mM}$ sodium phosphate, $\mathrm{pH}$ 7.2) or with partially purified rabbit or human antigen fractions in the same buffer. Preliminary experiments indicated that the plates were most efficiently 
coated by drying the antigen onto the plates overnight at $22^{\circ} \mathrm{C}$, and in most experiments, this procedure was used. The wells were washed and blocked with RPMI 1640 containing $10 \%$ fetal bovine serum for $1 \frac{1}{2} \mathrm{~h}$ at $22^{\circ} \mathrm{C}$. Sera were diluted in blocking medium and added to the coated/blocked wells for $2 \mathrm{~h}$ at $22^{\circ} \mathrm{C}$, then washed. The second antibody was peroxidase-conjugated goat anti-human light chain antibody ( $\alpha$ and $\lambda$ chain specificities; Tago Inc., Burlingame, CA) diluted 1:1500 in blocking medium. After incubating for $1 \mathrm{~h}$ with the peroxidaseconjugated antibodies, the wells were washed and aminoantipyrine/ phenol substrate (24) was added for $1 \frac{1}{2} \mathrm{~h}$ before reading absorbance at $490 \mathrm{~nm}$ in a Dynatech Microelisa Minireader (Dynatech Instruments, Santa Monica, CA).

Ion-exchange chromatography. All procedures were performed at $4^{\circ} \mathrm{C}$ in the presence of $0.1 \mathrm{mM}$ phenylmethylsulfonyl fluoride (PMSF) unless otherwise noted. Crude rabbit thymus extract was dialyzed into

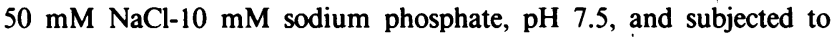
anion-exchange chromatography on DE-52 (Whatman Chemical Separation Inc., Clifton, $\mathrm{NJ}$ ). The column was eluted with a continuous gradient of $50-500 \mathrm{mM} \mathrm{NaCl}$ in the same buffer without PMSF as previously described (14). Fractions were assayed by ELISA for reactivity with anti-Sm and anti-RNP reference sera diluted 1:200 in blocking medium. The semiquantitative nature of the ELISA allowed the detection of specific peaks of antigen activity which reacted with anti$\mathrm{Sm}$, anti-RNP or both reference sera (see Results). The patterns of reactivity with the fractions obtained by using other sera containing unknown anti-ENA antibodies were then compared with the patterns obtained by using the anti-Sm and anti-RNP reference sera. This permitted the tentative identification of anti-Sm and/or anti-RNP antibodies in the sera (see Results). The presence of these antibodies in the sera was then confirmed by the radioimmunoprecipitation technique.

Radioimmunoprecipitation experiments. The presence of antibodies to Sm or RNP in sera was determined by immunoprecipitation of radiolabeled cell extracts followed by SDS-polyacrylamide gel electrophoresis (SDS-PAGE) of the radioactive proteins, as described (15). Briefly, cells were labeled for $12 \mathrm{~h}$ at $1.5-3 \times 10^{6}$ per $\mathrm{ml}$ in methioninedeficient medium containing $50 \mu \mathrm{Ci} / \mathrm{ml}$ of $\left[{ }^{35} \mathrm{~S}\right]$ methionine (New England Nuclear, Boston, MA; sp act $1,100 \mathrm{Ci} / \mathrm{mmol}$ ). The cells were washed, resuspended in $150 \mathrm{mM} \mathrm{NaCl}-10 \mathrm{mM}$ sodium phosphate, $\mathrm{pH}$ 7.2, containing $0.5 \mathrm{mM}$ PMSF-5 mM iodoacetamide- $0.3 \% \mathrm{NP}-40$, and sonicated for $20 \mathrm{~s}$ three times on ice using a Branson sonicator (Branson Sonic Power Co., Danbury, CT). The extract was centrifuged and $10 \mu \mathrm{l}$ of serum was added to $5 \times 10^{5}$ cell equivalents of extract for each precipitate. The precipitates were adsorbed to protein $A$ sepharose (Pharmacia Fine Chemicals, Piscataway, NJ), washed, eluted, and analyzed by SDS-PAGE (15). Human B cells (CESS) or erythroleukemia cells (K562) were used in most instances. In other experiments, $60 \times 10^{6}$ cells were harvested, sonicated on ice, and extracted in 150 $\mathrm{mM} \mathrm{NaCl}-30 \mathrm{mM}$ sodium phosphate, $\mathrm{pH}$ 7.5-0.5 mM PMSF-5 mM iodoacetamide. The sonicate was diluted to $50 \mathrm{mM} \mathrm{NaCl}-10 \mathrm{mM}$ sodium phosphate, centrifuged at $10,000 \mathrm{rpm}$ for $10 \mathrm{~min}$, and fractionated on DE-52 as above. Fractions were analyzed for Sm and RNP activity by ELISA, and $0.5 \mathrm{ml}$ of every third fraction was immunoprecipitated using $10 \mu \mathrm{l}$ of anti-Sm or anti-RNP reference sera followed by analysis on $15 \%$ SDS-polyacrylamide gels as above.

Competition assays. Sera were selected that contained predominantly anti-Sm and/or anti-RNP antibodies as verified by ELISA using DE-52 fractions and immunoprecipitation of ${ }^{35} \mathrm{~S}$-labeled cell extracts. For competition experiments, microtiter plates were coated with $50 \mu \mathrm{l}$ of a specific antigen fraction at $50 \mu \mathrm{g} / \mathrm{ml}$. The antigens consisted of pooled peaks of activity containing either $\mathrm{Sm}$ free of RNP activity or Sm plus RNP antigen, which had been obtained by ion-exchange chromatography on DE-52 (see above). The binding of various diluted sera to the antigen-coated wells was tested after preincubating the sera with the same or a different antigen fraction as follows: serum was diluted 1:200 or 1:400 in blocking medium and $100 \mu \mathrm{l}$ of the diluted serum was incubated with $50 \mu \mathrm{l}$ of serially diluted $\mathrm{Sm}$ or Sm plus RNP antigen in glass tubes for $2 \mathrm{~h}$ at $25^{\circ} \mathrm{C} ; 125 \mu \mathrm{l}$ of this mixture was then transferred into each antigen-coated well of a microtiter plate, and the ELISA was performed as above. Results were expressed as percent inhibition of total binding in the absence of competitor.

Enzyme sensitivity of the antigens. Antigens were treated for $1 \mathrm{~h}$ at $37^{\circ} \mathrm{C}$ either before or after coating onto ELISA plates with the following enzymes: pancreatic ribonuclease (Sigma Chemical Co., St. Louis, MO) $1 \mathrm{mg} / \mathrm{ml}$ in phosphate-buffered saline, $\mathrm{pH} 7.2$, snake venom exonuclease (Sigma Chemical Co., type IV, from Crotalus atrox) $1.5 \mathrm{mg} / \mathrm{ml}$ in $10 \mathrm{mM}$ Tris- $10 \mathrm{mM} \mathrm{NaCl}-5 \mathrm{mM} \mathrm{MgCl}$, $\mathrm{pH} 8.0$, and trypsin (Worthington Biochemical Corp., Freehold, NJ) $1 \mathrm{mg} / \mathrm{ml}$ in phosphate-buffered saline, $\mathrm{pH}$ 7.2. The relative amount of binding of antibody to untreated and enzyme-treated antigens in the ELISA was determined from standard curves of the optical density versus antibody dilution for each serum tested. Results were expressed as percent reduction in binding.

\section{Results}

The presence of autoantibodies against any of the multiple saline-extractable nuclear antigens present in either rabbit thymus extract or nuclear extracts from human cell lines (antiENA antibodies) was determined by ELISA. The dilution at which the binding of patient serum fell to the control value (normal human serum) was reported as the antibody titer. By this method, 42 of 50 sera $(84 \%)$ from patients with SLE displayed more antibody binding to ENA than control sera, as opposed to 17 of $50(34 \%)$ by passive hemagglutination (Table I). In general, the ELISA was 100-1,000 times more sensitive than the passive hemagglutination technique for detecting anti-ENA antibodies. This was due, in part, to the fact that antibodies to proteins other than Sm and RNP were also detected in this ELISA (see below). Antibodies to these additional proteins were usually not detectable by passive hemagglutination.

Separation of particles containing $\mathrm{Sm}$ from particles containing Sm plus RNP. To determine which of the sera containing anti-ENA antibodies also contained anti-Sm or RNP antibodies, the ribonucleoprotein complexes in rabbit thymus extract that contained Sm alone were separated from complexes containing both Sm and RNP by ion-exchange chromatography (Fig. 1). Antigen fractions were assayed by drying $50 \mu \mathrm{l}$ of each fraction onto a separate well of the microtiter plate followed by detection of specific antigens by ELISA using diluted anti-Sm or anti-RNP reference sera. Preliminary experiments showed that absorbance at $490 \mathrm{~nm}$ in the ELISA increased nearly linearly with the log of the antigen concentration over a wide range, allowing the relative amounts of antigen to be determined semiquantitatively by this direct binding assay.

Table I. Antibodies to ENAs in 50 SLE Sera

\begin{tabular}{lcc}
\hline Anti-ENA Titer & ELISA $^{*}$ & Hemagglutination \\
\hline Negative & 8 & 33 \\
$\leqq 1: 50$ & 6 & 3 \\
$1: 51-1: 500$ & 5 & 6 \\
$1: 501-1: 5000$ & 16 & 5 \\
$>1: 5000$ & 15 & 3
\end{tabular}

* Number of sera with given titer by ELISA.

$\ddagger$ Number of sera with given titer by passive hemagglutination (2). 


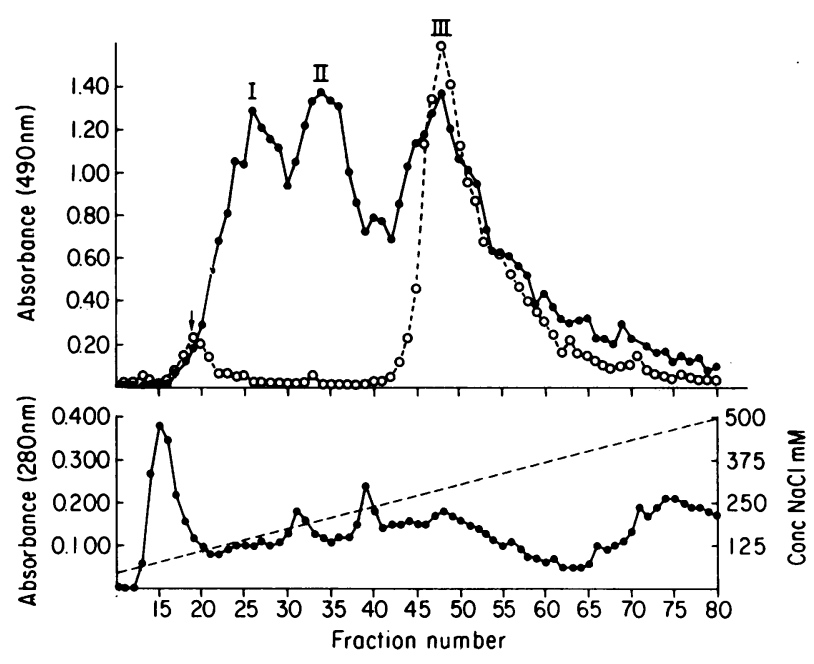

Figure 1. Separation of ribonucleoprotein complexes by anion-exchange chromatography. Rabbit thymus extract was adsorbed to DE52 in $50 \mathrm{mM} \mathrm{NaCl}-10 \mathrm{mM}$ sodium phosphate $\mathrm{pH} 7.5$ and eluted with a $200-\mathrm{ml}$ linear gradient of $50-500 \mathrm{mM} \mathrm{NaCl}$ in the same buffer, collecting $2.5-\mathrm{ml}$ fractions. Fractions were analyzed by ELISA for Sm and RNP antigen using 1:200 anti-Sm (•) or anti-RNP (O) reference sera (upper panel). Three peaks reactive with anti-Sm were obtained (I, II, and III). Anti-RNP serum reacted only with peak III. Note the additional peak of activity detected by the anti-RNP serum (arrow). This activity was not detected using other anti-RNP sera, and represents an antigen other than Sm or RNP, which is recognized by the patient's serum. Total protein (bottom panel) eluted as determined by absorbance at $280 \mathrm{~nm}$ is shown (๑) as well as the approximate concentration of $\mathrm{NaCl}$ in different fractions (-- -).

When rabbit thymus acetone extract was fractionated on DE-52, three major peaks of activity were consistently seen with anti-Sm reference sera, whereas anti-RNP reference sera detected a single peak of activity which coincided with the third peak detected by anti-Sm sera (Fig. 1). Thus, the first two peaks (I and II), eluting at $\sim 125$ and $150 \mathrm{mM} \mathrm{NaCl}$, contained Sm antigen free of detectable RNP activity, while the third peak (III), eluting at $\sim 260 \mathrm{mM} \mathrm{NaCl}$ contained both Sm and RNP antigens. Gel filtration experiments using an 80 $\times 1.5-\mathrm{cm}$ column of Sephacryl S300 (Pharmacia Fine Chemicals) indicated that the Sm and RNP activities in each of these peaks probably resided in particles rather than in the individual protein moieties, in that the mobilities of the antigens corresponded to $>70,000 \mathrm{~mol}$ wt (data not shown) as opposed to the $8,000-30,000 \mathrm{~mol}$ wt previously reported for the individual proteins $(5,15)$. The mobilities of the complexes on sucrose gradients also support this interpretation (not shown).

When Sm- and RNP-containing ribonucleoprotein particles from human cell extracts were separated by ion-exchange chromatography, somewhat different results were obtained (Fig. 2). As with rabbit thymus extract, a prominent peak of activity containing both Sm and RNP antigens was observed (Fig. 2, peak III). However the bulk of the Sm activity which was free of RNP activity eluted from DE-52 after peak III (Fig. 2, peak IV) rather than in peaks I and II. This fourth peak was barely detectable in commercial rabbit thymus extract (Fig. 1). Peaks I and II were always present in extracts from some human cell lines, such as CESS and K562 (Fig. 2),

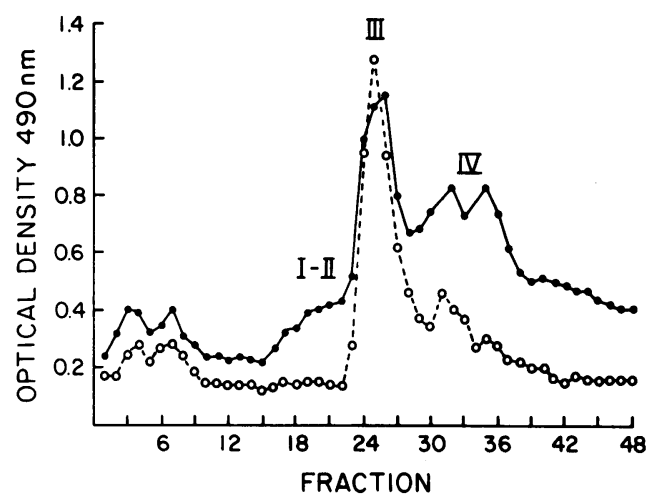

Figure 2. Separation of $\left[{ }^{35}\right.$ S $]$ methionine-labeled human ribonucleoprotein complexes by anion-exchange chromatography. Human B lymphoblastoid cells (CESS) were labeled for $12 \mathrm{~h}$ with

$\left[{ }^{35}\right.$ S $]$ methionine and an extract from $60 \times 10^{6}$ cells was adsorbed to DE-52 and eluted with a $100-\mathrm{ml}$ linear gradient of $50-500 \mathrm{mM} \mathrm{NaCl}$ in phosphate buffer collecting $2.0-\mathrm{ml}$ fractions. Sm and RNP antigenic activity was detected by ELISA using 1:200 anti-Sm (๑) or antiRNP (o) reference sera as in Fig. 1. Positions of peaks I-IV are indicated. The antigens in the fractions were also analyzed by immunoprecipitation using the same anti-Sm or RNP reference sera followed by SDS-PAGE of the precipitates (Fig. 3). In comparing Figs. 1 and 2, note that the rabbit thymus extract column (Fig. 1) was eluted with a $200-\mathrm{ml}$ gradient, and the human cell extract column (Fig. 2) was eluted with a 100 -ml gradient.

but in smaller amounts than in rabbit thymus extract (cf. Figs. 1 and 2). In other human cell lines, peaks I and II were completely absent (e.g., KE-37). To investigate further the differences in the patterns obtained by using commercial rabbit thymus extract and the human cell lines, extracts were prepared from homogenized fresh rabbit thymus by using the same procedure as used for preparation of the human cell extracts. When fresh rabbit thymus was used instead of the commercial rabbit thymus acetone powder, all four peaks of activity were detectable after fractionation on DE-52, and the pattern obtained was similar to that obtained using human CESS cells (data not shown; cf. Fig. 2). This finding is consistent with previous studies that used calf thymus (14).

The antigens reacting with anti-Sm and anti-RNP sera in these four peaks of activity were identified by metabolic labeling of human cells with $\left[{ }^{35} \mathrm{~S}\right]$ methionine followed by fractionation of a cell extract on DE-52. Sm and RNP activities were detected by ELISA (Fig. 2), and every third fraction was immunoprecipitated by using Sm or RNP reference sera followed by SDS-PAGE of the radioactive proteins. Both antiSm and anti-RNP reference sera specifically precipitated the same nine Sm and RNP proteins from peak III (Fig. 3, lane 1) that were not precipitated by normal serum (lane 2). AntiSm reference serum (Fig. 3, lane 3) but not anti-RNP or normal serum (lane 4 ) specifically precipitated bands $B, B^{\prime}, D$, $E, F$, and $G$ but not the RNP bands $A$ and $C$ from peak IV as well as from peaks I-II. The results shown in Fig. 3 are representative of those obtained using several reference sera.

Anti-Sm and RNP antibodies in autoimmune sera. By experiments similar to that illustrated in Fig. 1, sera containing either anti-Sm alone or anti-Sm plus anti-RNP antibodies (which react with peaks I-IV) could be distinguished from sera containing only anti-RNP antibodies (which react with 


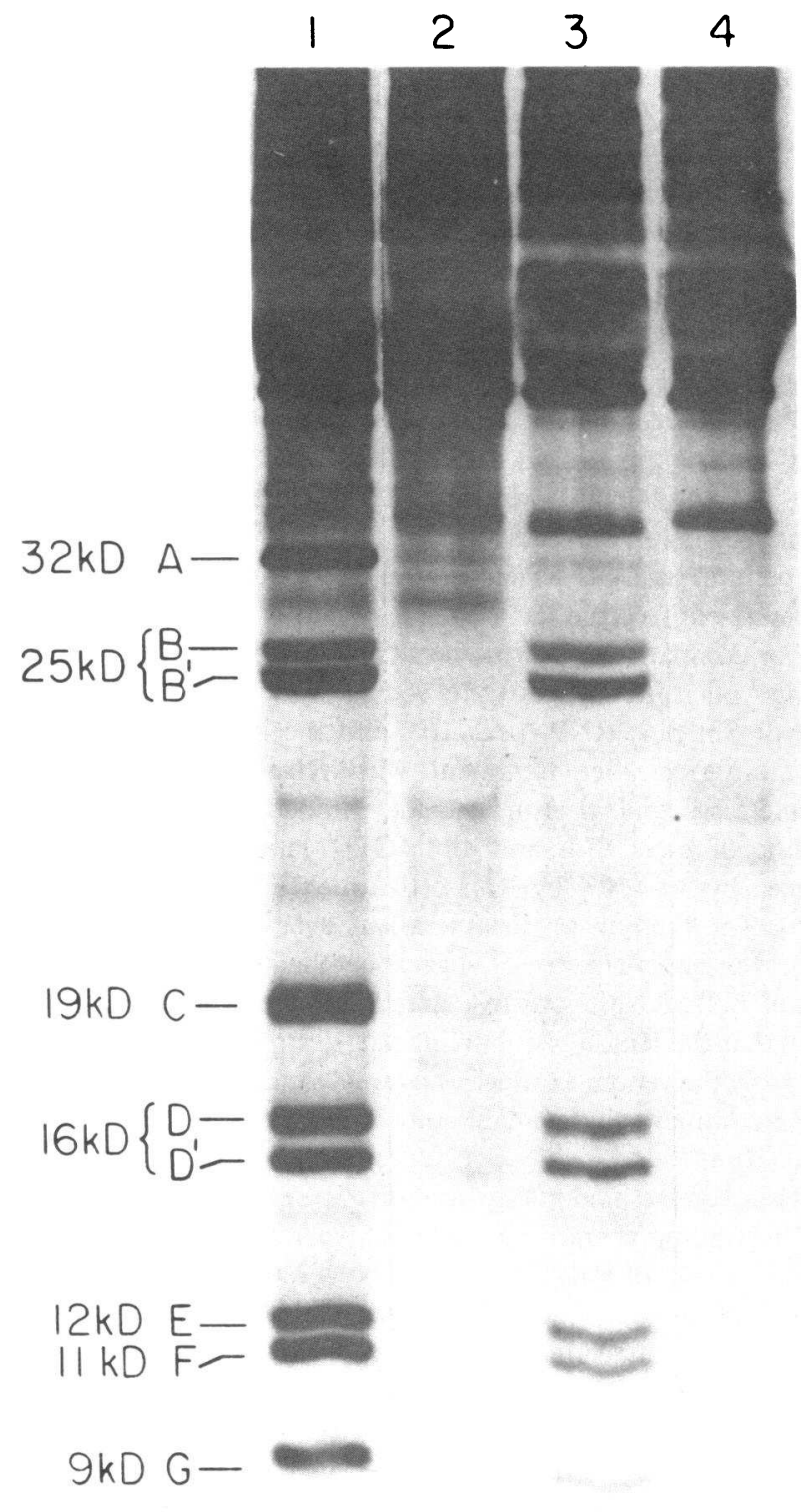

Figure 3. Immunoprecipitates of radiolabeled Sm and RNP ribonucleoprotein complexes separated by anion-exchange chromatography. Equivalent amounts of radioactive proteins from the experiment shown in Fig. 2 were immunoprecipitated using either anti-Sm (lanes 1 and 3) or normal (lanes 2 and 4 ) serum, followed by SDS-PAGE of the washed precipitates. Lane 1, peak III (fractions 24-27) precipitated with anti-Sm serum; lane 2, peak III with normal human serum; lane 3, peak IV (fractions 34-38) precipitated with anti-Sm; lane 4, peak IV with normal human serum. Positions and approximate molecular weights of the Sm and RNP proteins A-G are indicated.

peak III but not peaks I, II, or IV) (Table II). Sera containing these antibodies were tentatively identified by comparing the patterns of reactivity obtained by using "unknown" sera with those obtained using anti-Sm or RNP reference sera (see Fig. 1). Using this ELISA technique, 30 out of 50 SLE sera $(60 \%)$ contained either anti-Sm or anti-RNP antibodies as compared with 17 of $50(34 \%)$ by passive hemagglutination; 24 out of $50(48 \%)$ reacted with $\mathrm{Sm}$ which was free of RNP antigen
Table II. Sm and RNP Antibodies in 50 SLE Sera

\begin{tabular}{lllll}
\hline & & \multicolumn{2}{l}{ ELISA reactivity } & \\
\cline { 3 - 4 } $\begin{array}{l}\text { Anti-ENA titer } \\
\text { (ELISA) }\end{array}$ & $\begin{array}{l}\text { No. of sera } \\
\text { with titer }\end{array}$ & Peaks I-II* & Peak III & $\begin{array}{l}\text { Anti-Sm or RNP by } \\
\text { immunoprecipitation }\end{array}$ \\
\hline Negative & 8 & 0 & 0 & 1 \\
$\leqq 1: 50$ & 6 & 0 & 0 & 1 \\
1:51-1:500 & 5 & 1 & 3 & 3 \\
$1: 501-1: 5000$ & 16 & 9 & 13 & 12 \\
$>5000$ & 15 & 14 & 14 & 14 \\
\hline
\end{tabular}

* Sm antigen.

$\ddagger$ Sm plus RNP antigen.

$\S$ Determined by SDS-PAGE of immunoprecipitates of radiolabeled Sm and RNP antigens (see Methods).

(peaks I-II) as opposed to 13 of $50(26 \%)$ by passive hemagglutination. These figures are higher than those previously obtained by counterimmunoelectrophoresis, gel diffusion, or passive hemagglutination $(1,25-27)$. In some instances, sera that reacted with antigens other than Sm and RNP could be distinguished from sera that contained only anti-Sm or RNP antibodies by the presence of reactivity with additional peaks of activity eluting at different positions than the Sm and RNP peaks (for example, see Fig. 1, arrow).

The ELISA results correlated well with the results of immunoprecipitation studies using the same group of 50 SLE sera. When radiolabeled nuclear antigens were immunoprecipitated using these sera and analyzed on 15\% SDS-polyacrylamide gels, $62 \%$ of the 50 SLE sera were capable of specifically precipitating the characteristic Sm and RNP proteins ranging in molecular weight from 9,000 to 32,000 (Table II, last column). All but two of the sera that were positive by ELISA were also positive by radioimmunoprecipitation, whereas three sera were positive by the latter technique that were negative by ELISA.

Competition experiments. Although the relative binding of different sera to peaks I and II compared with that of peak III gave some indication of the relative amounts of anti-Sm and RNP antibodies in these sera, a more quantitative estimate could be obtained by competition experiments that use the separated Sm- and Sm plus RNP-containing ribonucleoprotein complexes. The fact that peaks I, II, and IV contained the same Sm proteins as peak III (Fig. 3) permitted the analysis of the reactivity of sera with the RNP-specific antigenic sites found in peak III. This was achieved by preadsorbing the sera with RNP-free Sm antigen obtained from peaks I-II (Fig. 1). Because the Sm and RNP antigens in peak III were not free of other contaminating antigens, the possibility of interference by contaminants in the competition assays was minimized in several ways. First, sera that reacted only with Sm and RNP antigens by gel diffusion and by immunoprecipitation of $\left[{ }^{35}\right.$ S]methionine-labeled nuclear antigens were selected for this analysis. Secondly, all sera were diluted 1:200 to 1:400 for the competition experiments to further minimize binding to contaminants by other autoantibodies present in low titers.

When sera were preincubated with $\mathrm{Sm}$ antigen (peaks I-II), antibody binding to the same antigen on the microtiter plate was reduced to background. Similarly, preincubation with Sm plus RNP (peak III) completely blocked binding to both peak III and peaks I-II on the microtiter plate. By coating 


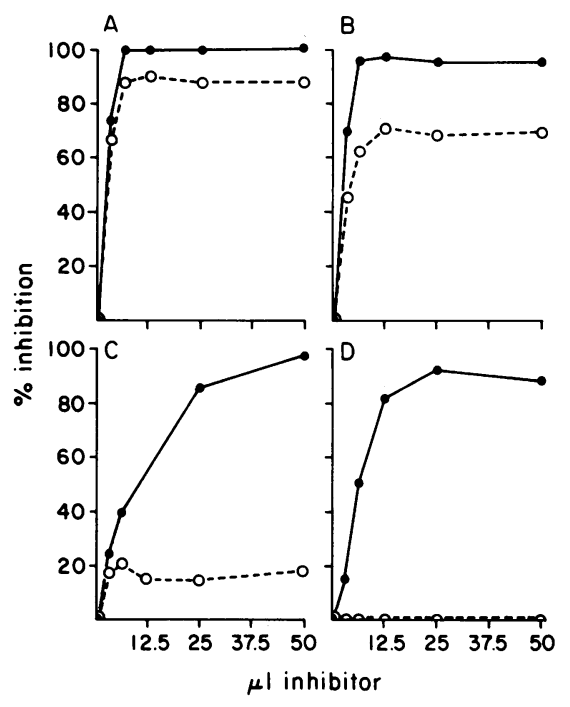

Figure 4. Competition experiments. Wells of microtiter plates were coated with $50 \mu \mathrm{g} / \mathrm{ml}$ of antigen from peak III (containing Sm plus RNP activity-see Fig. 1). Patient serum was preincubated with different amounts of the same antigen at $500 \mu \mathrm{g} / \mathrm{ml}(\bullet)$ or with $\mathrm{Sm}$ antigen $(500 \mu \mathrm{g} / \mathrm{ml})$ from peaks I-II (O) before binding to the coated wells. Results are expressed as percent inhibition of the binding of serum in the absence of competition. $(A)$ Patient PM with SLE, serum diluted 1:200, $(B)$ patient GK with SLE, serum diluted 1:200; $(C)$ patient PP with SLE, serum diluted 1:200; $(D)$ patient JM with MCTD, serum diluted 1:400.

the plate with antigen from peak III and preadsorbing the sera with Sm antigen from peaks I-II, an estimate of the amount of binding to antigenic sites present exclusively in peak III ("RNP-like antibody") was made (Fig. 4, $A-D$ ). In Fig. 4, $A-C$ illustrate the results obtained by using SLE sera in which $\sim 90 \%, 70 \%$, and $15 \%$ of the binding to peak III could be blocked by preincubation with Sm antigen. 21 of the 24 SLE sera analyzed gave a pattern similar to that shown in Fig. 4
C. Fig. $4 \mathrm{D}$ illustrates the results of the same experiment using a MCTD serum, which contained no anti-Sm. Of the SLE sera examined by this technique, 3 of 24 contained antibodies whose binding to peak III was $>50 \%$ inhibited by preincubation with Sm, indicating that only a small minority of SLE sera contined a larger amount of anti-Sm reactivity than anti-RNP reactivity. In the other 21 sera, the binding to peak III appeared to be mainly due to anti-RNP antibodies. In no instance was all of the binding to peak III inhibited by preincubation with Sm, suggesting that all 24 lupus sera contained antibodies to RNP-like antigenic sites. In contrast, the binding of seven of eight MCTD sera to peak III was unaffected by preincubation with Sm, in agreement with findings of others that these sera do not generally contain anti-Sm $(1,11,12)$. The results of competition analysis using selected SLE and MCTD sera are summarized in Table III.

For comparison with the results of the competition experiments, microtiter plates were coated with either Sm (peaks I-II) or Sm plus RNP (peak III), and the binding of sera to these antigens after treatment with RNase, snake venom exonuclease, and trypsin was determined (Table II). The binding of most SLE and all MCTD sera to peak III was reduced by $80-100 \%$ by RNase treatment (Table III, patients 4-15). The binding of these sera was even more sensitive to snake venom exonuclease, suggesting that this enzyme may disrupt both RNase-sensitive and RNase-resistant sites. It is likely that the Rnase-resistant site was destroyed by enzymatic attack (by the venom exonuclease) on RNA, although impurities in the commercially available enzyme (e.g., protease) cannot be ruled out.

The decrease in antibody binding to peak III after treatment with RNase or venom exonuclease generally correlated with the percentage of anti-RNP antibody as estimated by competition experiments (Table III). In several SLE sera, however, the RNase-sensitive binding correlated well with the percentage of RNP-like antibody as determined by competition experiments, but this binding was completely resistant to venom exonuclease (Table III, patients 1-3). This may represent

Table III. Estimation of Relative Amounts Anti-Sm and RNP Antibodies in Selected Sera

\begin{tabular}{|c|c|c|c|c|c|c|c|c|}
\hline \multirow[b]{2}{*}{ No. } & \multirow[b]{2}{*}{ Patient } & \multirow[b]{2}{*}{ Diagnosis } & \multirow[b]{2}{*}{ Tótal ENA titer } & \multirow[b]{2}{*}{$\% \mathrm{Sm}^{*}$} & \multirow[b]{2}{*}{ \% RNPł } & \multicolumn{3}{|c|}{ \% Decrease binding $\$$} \\
\hline & & & & & & RNase & SVE & Trypsin \\
\hline 1 & PM & SLE & $1: 5,000$ & 90 & 10 & 22 & 0 & 50 \\
\hline 2 & DJo & SLE & $1: 25,000$ & 85 & 15 & 32 & 0 & 69 \\
\hline 3 & GK & SLE & $1: 25,000$ & 70 & 30 & 38 & 0 & 58 \\
\hline 4 & SO & SLE & $1: 3,125,000$ & 20 & 80 & 89 & 83 & $\sim 90$ \\
\hline 5 & PP & SLE & $1: 25,000$ & 15 & 85 & 79 & 85 & $\sim 80$ \\
\hline 6 & RT & SLE & $1: 625,000$ & 15 & 85 & 83 & 93 & $\sim 100$ \\
\hline 7 & $\mathrm{CT}$ & SLE & $1: 25,000$ & 15 & 85 & 33 & 50 & 56 \\
\hline 8 & MY & SLE & $1: 5,000$ & 10 & 90 & 80 & 83 & $\sim 100$ \\
\hline 9 & PHy & SLE & $1: 625,000$ & 10 & 90 & 89 & 89 & $\sim 90$ \\
\hline 10 & DS & SLE & $1: 125,000$ & 5 & 95 & 73 & 85 & 71 \\
\hline 11 & $\mathrm{DJa}$ & MCTD & $1: 125,000$ & $\sim 0$ & $\sim 100$ & 84 & 96 & $\sim 100$ \\
\hline 12 & PHu & MCTD & $1: 125,000$ & 0 & 100 & 81 & 93 & $\sim 100$ \\
\hline 13 & $\mathrm{JM}$ & MCTD & $1: 125,000$ & 0 & 100 & 82 & 95 & $\sim 100$ \\
\hline 14 & KA & MCTD & $1: 3,125,000$ & 0 & 100 & 94 & 99 & $\sim 100$ \\
\hline 15 & SL & MCTD & $1: 625,000$ & 0 & 100 & $>95$ & $>95$ & $\sim 100$ \\
\hline
\end{tabular}

* Percent of binding to peak III in competition experiments that was inhibitable by preadsorption with Sm antigen. $¥$ Percent of binding to peak III in competition experiments that could not be inhibited by preadsorption with Sm antigen. § Percent decrease in antibody binding to peak III by ELISA after digestion of the antigen with ribonuclease (RNase), snake venom exonuclease (SVE), or trypsin (see Methods). 
binding to an additional determinant which is present only on ribonucleoprotein particles containing both $\mathrm{Sm}$ and RNP. Serum from patient 7 in Table III appeared to react with this antigenic site as well as with the other RNP determinants. The binding of sera containing anti-Sm to $\mathrm{Sm}$ antigen was also partially sensitive to RNase but was mostly resistant to venom exonuclease (data not shown).

\section{Discussion}

In the experiments described above, the magnitude of the humoral immune response to various antigenic sites on Smand RNP-containing ribonucleoprotein particles in SLE and MCTD was studied. The binding of antibodies to these antigenic sites was determined by methods that were not dependent upon the sensitivity of the antigens to ribonuclease. This is important in view of studies by White and colleagues (19-21), who suggest that RNP is partly resistant and Sm partly sensitive to RNase treatment. Attempts to circumvent this problem have been only partly successful. For example, analysis of the RNA species immunoprecipitated by various patient sera (28) can distinguish between sera containing anti-RNP antibodies alone (which precipitate only U1-RNA) and sera containing either anti-Sm alone or anti-Sm plus anti-RNP (which precipitate U1, U2, and U4-6 RNAs). However, antiSm cannot be distinguished from anti-Sm plus anti-RNP by this method because of the physical association of both $\mathrm{Sm}$ and RNP antigens with U1 RNA (5). Western blotting has also been partially successful in resolving this problem (15, 29-31) but has been hampered by the fact that only those antigenic sites that are not destroyed by SDS and are independent of an association with RNA are readily detectable.

The competition experiments presented here circumvent these problems by defining as "RNP-like" those antibodies that react specifically with U1-RNP particles (i.e., peak III, Figs. 1 and 2). That peak III contains U1-RNP articles is virtually certain based on the finding that the U1-RNP-specific $(5,15,16,17,29)$ proteins $A$ and $C$ are found only in this peak of activity (Fig. 3). Thus, RNP-like antibodies include antibodies that react with proteins $\mathrm{A}$ and $\mathrm{C}$, either alone or in combination with U1-RNA, regardless of whether or not the antigenic sites are sensitive to RNase. In addition, RNP-like antibodies would include antibodies to the higher molecular weight proteins that reportedly are specifically attached to U1-RNP particles $(21,29)$, although we have not consistently detected such proteins.

The competition experiments have allowed sera containing antibodies to Sm or RNP to be divided into three somewhat arbitrary groups, based on their reactivity with separated ribonucleoprotein particles. The first group (Table 3, patients 11-15) consists of MCTD sera, which reacted only with peak III. These sera recognized mainly a RNase-sensitive site but also recognized a RNase-resistant site, which was sensitive to snake venom exonuclease. Although a portion of the binding of MCTD sera to peak III was insensitive to RNase treatment of the antigen (and would previously have been defined "Sm" antibody), all of the binding was apparently U1-RNP specific (see Figs. 1 and 4 and Table 3) or RNP-like. Previous studies from our laboratory have indicated that MCTD sera react with two proteins, bands $A$ and $C$, one of which (band $A$ ) is released from the U1-RNP article in an immunologically unreactive form after treatment with RNase $(17,32,33)$. Band C, however, remains reactive with MCTD sera after RNase treatment. Antibody against band $\mathrm{C}$ may therefore be responsible for the portion of the binding that was RNase-resistant and venom exonuclease-sensitive.

The second group consists of SLE sera that reacted primarily with the same antigenic sites as recognized by MCTD sera. This group was composed of 21 of the 24 SLE sera that had either anti-Sm or RNP antibodies. In these 21 sera, $<20 \%$ of the antibody binding to peak III could be inhibited by preincubation with Sm antigen (see Table III, patients 4-10). The RNase and snake venom exonuclease sensitivity of the remaining $80 \%$ or more of the binding supported the interpretation that this portion of the activity represented binding to the same sites recognized by the MCTD sera.

In the third group, consisting of 3 of the 24 SLE sera, most of the binding to peak III was attributable to the presence of antibodies against shared "Sm" determinants. However, each of these sera contained additional antibodies whose binding to peak III could not be inhibited by pre-incubation with $\mathrm{Sm}$. The determinant recognized by these sera is apparently different from those recognized by MCTD sera and the other 21 SLE sera, because although the binding to peak III was partially sensitive to RNase, it was completely resistant to snake venom exonuclease (Table III, patients 1-3). Although the possibility exists that this additional activity is due to binding to a contaminating ribonucleoprotein other than Sm or RNP, we feel this is unlikely, because when these sera were preadsorbed with $\mathrm{Sm}$, they no longer immunoprecipitated radiolabeled Sm complexes, but still were capable of immunoprecipitating complexes containing both Sm and RNP from peak III (unpublished observation). Further characterization of this additional RNP-like determinant is in progress.

A fourth group of sera could theoretically be distinguished by experiments such as that illustrated in Figs. 1 and 2 . These sera would contain antibodies specific for $\mathrm{U} 2, \mathrm{U} 4, \mathrm{U} 5$, or $\mathrm{U} 6$ particles, and might be expected to react with peaks I, II, or IV but not with peak III. Although no such sera were identified in the present studies, antibodies which react specifically with U2 particles have recently been identified by others in sera from patients with scleroderma-polymyositis overlap syndrome (34).

These studies indicate that most SLE and all MCTD sera that contain Sm or RNP antibodies contain an overwhelming preponderance of antibodies, which are apparently specifically reactive with U1-RNP particles, and that antibodies against the Sm determinants, which are shared by U1, U2, and U4-6 particles, represent a minor component of the total humoral immune response to these particles. This might reflect merely the relative accessibility of the various sites to immune attack. Alternatively, it might suggest that U1-RNP particles are especially immunogenic in these patients, either because of some characteristic of the particle itself or because of increased amounts of U1-RNP particles relative to U2, U4, U5, or U6 particles. It should be noted that the U1-RNP particle is the most prevalent form of Sm and RNP antigen in calf thymus (10) as well as in all of the human cell lines we have studied (see Fig. 2). Also, recent studies demonstrate that RNP antigen (presumably in the form of U1-RNP particles) is induced in ultraviolet-irradiated keratinocytes (35), and preliminary experiments in our laboratory suggest that synthesis of this particle is also increased in mitogen-stimulated murine B 
lymphocytes. The preferential synthesis of U1-RNP particles in response to certain external stimuli might provide a mechanism whereby the antigen load of this particular type of ribonucleoprotein particle is transiently increased, permitting the production of autoantibodies against it. Such a mechanism has been previously postulated to play a role in other examples of autoimmunity $(36,37)$.

In addition to the striking predominance of the U1-RNP peak of activity detected in human cell lines (see Fig. 2, peak III), human cell extracts differed from rabbit thymus acetone extract in the elution pattern of peaks I, II, and IV containing Sm antigens (cf. Figs. 1 and 2). The prominence of peaks I and II compared to peak IV in rabbit thymus extract and the prominence of peak IV in human cell extracts might be explained in several ways. The patterns obtained might be characteristic of a given species or cell type. Alternatively, the different patterns may be related to the method used to prepare the antigens (e.g., acetone precipitation in the case of the commercially available rabbit thymus acetone powder). The experiments using fresh rabbit thymus instead of the commercial rabbit thymus acetone powder suggest that both of these alternatives may be correct. Large amounts of $\mathrm{Sm}$ activity which eluted at high salt (peak IV) were obtained after fractionation of fresh rabbit thymus extract on DE-52, suggesting that the near absence of peak IV in commercial rabbit thymus acetone powder (Fig. 1) is probably an artifact of the organic solvent fractionation procedure. Possibly the lower polarity of acetone reduces hydrophobic forces within the Sm complexes in peak IV, resulting in their partial (or complete) dissociation. In contrast, the presence of relatively large amounts of peaks I-II in both preparations of rabbit thymus extract and in fresh calf thymus extract (14) suggests that thymus may be enriched in this form of Sm compared to other tissue or cell types.

The reason that some Sm eluted at low (125-150 mM) salt whereas some other Sm eluted at high (>260 mM) salt (see Figs. 1 and 2) is of some interest, in that the protein bands in peaks I-II and IV were the same. This implies either a difference in a protein(s) which is/are not labelled by $\left[{ }^{35}\right.$ S $]$ methionine or, more likely, a difference in the RNA present. We are currently investigating the possibility that peaks I-II contain Sm particles that have not yet bound to RNA or which have dissociated from RNA. Recent studies from our laboratory have demonstrated the existence of a RNA-free 6S precursor article, which contains several of the Sm proteins and which is subsequently assembled into mature 10S ribonucleoprotein particles (manuscript submitted for publication). The relationship of the $6 \mathrm{~S}$ particle to the DE-52 elution pattern remains to be clarified, but the RNA-free particle might be expected to elute from the ion-exchange matrix at a significantly lower salt concentration than the mature ribonucleoprotein particles due to the high negative charge density of RNA.

The present studies indicate that both the ELISA using DE-52 fractions and the radioimmunoprecipitation techniques are significantly more sensitive than passive hemagglutination and other standard techniques for detecting Sm and RNP antibodies, and that the sensitivity of the ELISA and radioimmunoprecipitation techniques is comparable. Approximately $60 \%$ of the SLE sera tested contained either Sm or RNP antibodies by both ELISA and immunoprecipitation, and at least $48 \%$ contained $\mathrm{Sm}$ antibodies by ELISA. In comparison,
$20-46 \%$ of SLE sera have been reported to contain anti-RNP antibodies by counterimmunoelectrophoresis, gel diffusion, or passive hemagglutination (1, 25-27), whereas 23-24\% of SLE sera have been shown to contain anti-Sm antibodies by counterimmunoelectrophoresis $(26,27)$. It is unclear at present whether or not those SLE sera that contained only RNP antibodies by ELISA might also contain small amounts of Sm antibodies which were undetectable by this technique.

It should be mentioned that the presence of "anti-ENA" antibodies in the screening ELISA does not necessariy indicate the presence of anti-Sm or anti-RNP antibodies. Although high titers of anti-ENA antibodies $(\geq 1: 5,000)$ correlated strongly with the presence of anti-Sm or anti-RNP antibodies (18 out of 19 instances), 12 out of the 50 sera reported here reacted with the unfractionated ENA, but failed to react with DE-52 peaks I-IV or to immunoprecipitate the $\left[{ }^{35}\right.$ S]methioninelabeled Sm and RNP antigens. These 12 sera had relatively low titers $(<1: 5000)$ of anti-ENA antibodies, and reacted with proteins other than Sm and RNP by the radioimmunoprecipitation technique. Obviously, the most reliable results are those obtained by radioimmunoprecipitation, but we have found that reactivity with specific peaks of activity by ELISA is nearly as good. This is especially true in the case of sera that contain anti-Sm antibodies, because these sera react with four characteristic peaks by ELISA (Figs. 1 and 2).

In summary, these studies indicate that a humoral immune response to Sm and RNP antigens exists in the majority of SLE and MCTD patients, and that in both diseases, this response is directed mainly against several different antigenic sites that are exclusive to U1-RNP particles. The production of anti-Sm antibodies in SLE appears to be quantitatively less prominent than the production of U1-RNP specific antibodies. These observations as well as the observation that extremely high titers of RNP antibodies are sometimes observed in the absence of other autoantibodies (reference 1 and present studies) would seem to favor a role of the U1-RNP antigen in producing this autoimmune response. Further studies will be needed in order to determine whether or not alterations in nuclear metabolism might play a role in production of antinuclear antibodies, or whether these antibodies result from a primary defect in immunoregulation (e.g., polyclonal activation).

\section{Acknowledgments}

The authors thank Dr. Günter Blobel for useful discussions after the untimely death of Dr. Kunkel. We also thank Dr. Nicholas Chiorazzi and Dr. Lloyd Mayer for critical review of the manuscript, and Elena Kissin and Rosanne Wisniewolski for technical assistance.

These studies were supported in part by grants AM-04761 (to Dr. Kunkel) and RR-00102 (to the Rockefeller University Hospital) from the National Institutes of Health, and by a grant from the Markey Trust. Dr. Reeves is a Fellow of the Arthritis Foundation.

\section{References}

1. Tan, E. M. 1982. Autoantibodies to nuclear antigens (ANA): their immunobiology and medicine. Adv. Immunol. 33:167-240.

2. Tan, E. M., and C. Peebles. 1976. In Manual of Clinical Immunology. N. R. Rose and H. Friedman, editors. American Society for Microbiology Press, Washington, DC. 660-664.

3. Douvas, A. S., M. Achten, and E. M. Tan. 1979. Identification of a nuclear protein (Scl-70) as a unique target of human antinuclear antibodies in scleroderma. J. Biol. Chem. 254:10514-10522. 
4. Bernstein, R. M., and M. B. Matthews. 1983. Myositis autoantibody inhibits histidyl-tRNA synthetase: a model for autoimmunity. Nature (Lond.). 304:177-179.

5. Lerner, M. R., and J. A. Steitz. 1979. Antibodies to small nuclear RNAs complexed with proteins are produced by patients with systemic lupus erythematosus. Proc. Natl. Acad. Sci. USA. 76:54955499.

6. Lerner, M. R., J. A. Boyle, J. A. Hardin, and J. A. Steitz. 1980. Are snRNP's involved in splicing? Nature (Lond.). 283:220-224.

7. Yang, W. V., M. R. Lerner, J. A. Steitz, and S. J. Flint. 1981. A small nuclear ribonucleoprotein is required for splicing of adenoviral early RNA sequences. Proc. Natl. Acad. Sci. USA. 78:1371-1375.

8. Lerner, M. R., N. C. Andrews, G. Miller, and J. A. Steitz. 1981. Two small RNA's encoded by Epstein-Barr virus and complexed with protein and precipitated by antibodies from patients with systemic lupus erythematosus. Proc. Natl. Acad. Sci. USA. 78:805-809.

9. Francoeur, A. M., and M. B. Matthews. 1982. Interaction between VA RNA and the lupus antigen La: formation of a ribonucleoprotein particle in vitro. Proc. Natl. Acad. Sci. USA. 79:67726776.

10. Mattioli, M., and M. Reichlin. 1973. Physical association of two nuclear antigens and mutual occurrence of their antibodies: the relationship of the Sm and RNA protein (Mo) systems in SLE sera. $J$. Immunol. 110:1318-1324.

11. Sharp, G. C., W. S. Irvis, E. M. Tan, R. G. Gould, and H. R. Holman. 1972. Mixed connective tissue disease: an apparently distinct rheumatic disease syndrome associated with a specific antibody to an extractable nuclear antigen (ENA). Am. J. Med. 52:148-159.

12. Sharp, G. C., W. S. Irvin, C. M. May, H. R. Holman, F. C. McDuffie, E. V. Hess, and F. R. Schmid. 1976. Association of antibodies to ribonucleoprotein and $\mathrm{Sm}$ antigens with mixed connective disease, systemic lupus erythematosus and other rheumatic diseases. N. Engl. J. Med. 295:1149-1154.

13. Mattioli, M., and M. Reichlin. 1971. Characterization of a soluble nuclear ribonucleoprotein antigen reactive with SLE sera. $J$. Immunol. 107:1281-1290.

14. Northway, J.' D., and E. M. Tan. 1972. Differentiation of antinuclear antibodies giving speckled staining patterns in immunofluơrescence. Clin. Immunol. Immunopathol. 1:140-154.

15. Conner, G. E., D. Nelson, R. Wisniewolski, R. G. Lahita, G. Blobel, and H. G. Kunkel. 1982. Protein antigens of the RNA-protein complexes detected by anti-Sm and anti-RNP antibodies found in serum of patients with systemic lupus erythematosus and related disorders. J. Exp. Med. 156:1475-1485.

16. Hinterberger, M., I. Petterson, and J. A. Steitz. 1983. Isolation of small nuclear ribonucleoproteins containing $\mathrm{U} 1, \mathrm{U} 2, \mathrm{U} 4, \mathrm{U} 5$ and U6 RNA's. J. Biol. Chem. 258:2604-2613.

17. Fisher, D. E., G. E. Conner, W. H. Reeves, G. Blobel, and H. G. Kunkel. 1983. Synthesis and assembly of human small nuclear ribonucleoproteins generated by cell free translation. Proc. Natl. Acad. Sci. USA. 80:6356-6360.

18. Moore, T. L., T. D. Weiss, S. H. Neuchs, A. R. Baldassare, and J. Zuckner. 1981. Extractable nuclear antigens. Semin. Arthritis Rheum. 10:309-318.

19. White, P. J., W. D. Gardner, and S. O. Hoch. 1981. Identification of the immunogenically active components of the Sm and RNP antigens. Proc. Natl. Acad. Sci. USA. 78:626-630.

20. White, P. J., P. B. Billings, and S. O. Hoch. 1982. Assays for the Sm and RNP autoantigens: the requirement for RNA and influence of the tissue source. J. Immunol. 128:1751-2756.

21. White, P. J., and S. O. Hoch. 1981. Definition of the antigenic polypeptides in the Sm and RNP ribonucleoprotein complexes. Biochem. Biophys. Res. Commun. 102:365-371.

22. Tan, E. M., A. S. Cohen, J. F. Fries, A. T. Masi, D. J. McShane, N. F. Rothfield, J. G. Schaller, N. Talal, and R. J. Winchester. 1982. The 1982 revised criteria for the classification of systemic lupus erythematosus. Arthritis Rheum. 25:1271-1277.

23. Yanker, B. A., and E. M. Shooter. 1979. Nerve growth factor in the nucleus: interaction with receptors on the nuclear membrane. Proc. Natl. Acad. Sci. USA. 76:1269-1273.

24. Giallongo, A., L. Kochoumian, and T. P. King. 1982. Enzyme and radioimmunoassays for specific murine $\operatorname{IgE}$ and $\operatorname{IgG}$ with different solid phase immunosorbants. J. Immunol. Methods. 52:379-393.

25. Schur, P. H., I. Meyer, M. Garovoy, and C. B. Carpenter. 1982. Associations between systemic lupus erythematosus and the major histocompatibility complex: clinical and immunological considerations. Clin. Immunol. Immunopathol. 24:263-275.

26. Kurata, N., and E. M. Tan. 1976. Identification of antibodies to nuclear acidic antigens by counterimmunoelectrophoresis. Arthritis Rheum. 19:574-580.

27. Keiser, H. D., and J. Weinstein. 1980. The detection and identification of antibodies to saline extractable nuclear antigens by counterimmunoelectrophoresis. Arthritis Rheum. 23:1026-1035.

28. Williamson, G. G., J. Pennebaker, and J. A. Boyle. 1983. Clinical characteristics of patients with rheumatic disorders who possess antibodies against ribonucleoprotein particles. Arthritis Rheum. 26: 509-515.

29. Kinlaw, C. S., B. L. Robberson, and S. M. Berget. 1983. Fractionation and characterization of human small nuclear ribonucleoproteins containing U1 and U2 RNAs. J. Biol. Chem. 258:7181-7189.

30. Pettersson, I., M. Hinterberger, M. Mimori, E. Gottlieb, and J. A. Steitz. 1984. The structure of mammalian small nuclear ribonucleoproteins: identification of multiple protein components reactive with anti(U1) RNP and anti-Sm antibodies. J. Biol. Chem. 259:59075914.

31. Habets, W. J., D. J. De Rooij, M. H. Salden, A. P. Verhagen, C. A. Van Eekelen, L. B. Van DePutte, and W. J. Van Venrooij. 1983. Antibodies against distinct nuclear matrix proteins are characteristic for mixed connective tissue disease. Clin. Exp. Immunol. 54: 265-276.

32. Fisher, D. E., G. E. Conner, W. H. Reeves, and H. G. Kunkel. 1983. Sm and RNP protein antigens are distinguishable by in vitro translation and pulse chase analysis. Arthritis Rheum. 26:S74.

33. Fisher, D. E., W. H. Reeves, G. E. Conner, G. Blobel, and H. G. Kunkel. 1984. Pulse labelling of small nuclear ribonucleoproteins in vivo reveals distinct patterns of antigen recognition by human autoimmune antibodies. Proc. Natl. Acad. Sci. USA. 81:3185-3189.

34. Mimori, T., M. Hinterberger, I. Pettersson, and J. A. Steitz. 1984. Autoantibodies to the $U 2$ small nuclear ribonucleoprotein in a patient with scleroderma-polymyositis overlap syndrome. J. Biol. Chem. 259:560-565.

35. LeFeber, W. P., D. A. Norris, J. C. Huff, M. Kubo, S. R. Ryan, and W. L. Weston. 1983. Induction of nuclear antigen expression in human keratinocytes by ultraviolet light (UVL). J. Invest. Dermatol. 80:328.

36. Weigle, W. O. 1981. Self-nonself recognition by $T$ and $B$ lymphocytes and their roles in autoimmune phenomena. Arthritis Rheum. 24:1044-1052.

37. Okayasee, I., and S. Hatakeyama. 1983. The difference in susceptibility of target thyroid gland to autoimmune thyroiditis induced by a combination of implantation of thyroid gland and injection of lipopolysaccharide. Clin. Immunol. Immunopathol. 29:51-57. 